\title{
The Effects of Smartphone, Tablet and Computer Overuse on Children's Eyes During the COVID-19 Pandemic
}

\author{
๑ Hakan Öztürk, ๑ Bediz Özen \\ University of Health Sciences Turkey, Tepecik Training and Research Hospital, Clinic of Ophthalmology, İzmir, Turkey
}

\begin{abstract}
Aim: Due to restrictions during the Coronavirus disease-2019 (COVID-19) pandemic, smartphone, tablet and computer (STC) overuse might occur. There were some concerns that light sources which emit blue light might affect anterior and posterior segment structures. We investigated the effects of STC overuse on children's eyes during the pandemic.

Materials and Methods: Sixty-four children's findings at baseline (the early period of the pandemic) (group 1) were compared with those after 1 year (group 2). Correlations between daily STC use time (DSTCUT) and the ocular parameters in group 2 were evaluated.

Results: Compared to group 1, group 2 had longer DSTCUT (1.49 \pm 0.33 vs $5.62 \pm 0.57$ hours, $p<0.001)$, lower tear break-up time (TBUT) (12.70 \pm 1.45 vs $9.50 \pm 2.10$ seconds, $p=0.015)$, higher ocular surface disease index (OSDI) score (14.52 \pm 4.19 vs $25.22 \pm 5.75, p=0.007)$, more myopic spherical equivalent refraction (SER) $(-1.00 \pm 0.30$ vs $-1.80 \pm 0.40$ dioptres, $p=0.031)$, and greater axial length $(22.69 \pm 0.43$ vs $23.42 \pm 0.37$ millimetres, $p=0.038)$. Schirmer tests, central corneal thicknesses $(C C T)$, keratometries, anterior chamber depths (ACD), lens thicknesses (LT), retinal nerve fibre layer thicknesses, choroidal thicknesses and macular thicknesses of groups were similar ( $p>0.05)$. In group 2 as DSTCUT increased, TBUT $(r=-0.368, p=0.034)$ decreased, OSDI score $(r=0.384, p=0.028)$ increased and more myopic SER $(r=0.340, p=0.045)$ occurred.

Conclusion: To our knowledge, this is the first study in children to comprehensively investigate the effects of STC overuse during a pandemic on ocular surface, anterior and posterior segment structures together. We found that STC overuse during the pandemic might increase the tendency to dry-eye and myopia, and might cause axial elongation. We also determined that at 1-year follow-up, STC overuse had no effect on $\mathrm{CCT}$, keratometry, ACD, LT, and posterior segment parameters.
\end{abstract}

Keywords: Smartphone, tablet, computer, children, eye, COVID-19 pandemic

\section{Introduction}

In children, the use of digital devices with a close-working distance, such as smartphones, tablets or computers (STC), has increased with their technological developments (1). These devices allow various activities, such as surfing the internet, playing games or drawing. Due to the restrictions during the Coronavirus disease-2019 (COVID-19) pandemic, the implementation of digital or e-learning approaches, instead of face-to-face education, has led to children being more likely to use STC. Moreover, as the time spent at home has increased during the pandemic, parents may give these devices to children more often to keep them busy. Therefore, as the duration of the pandemic extends, children might start to overuse these devices in daily life. The American Academy of Pediatrics recommends that daily screen use in children aged 5-18 years should not exceed 2 hours (2). The

\section{Address for Correspondence}

Bediz Özen, University of Health Sciences Turkey, Tepecik Training and Research Hospital, Clinic of Ophthalmology, Izmir, Turkey Phone: +90 5053767386 E-mail: bedizozen@yahoo.com ORCID: orcid.org/0000-0001-9020-3810 Received: 17.06.2021 Accepted: 06.07.2021

${ }^{\circ}$ Copyright 2021 by Ege University Faculty of Medicine, Department of Pediatrics and Ege Children's Foundation The Journal of Pediatric Research, published by Galenos Publishing House. 
COVID-19 pandemic can place a significant burden on health and the economy. Prolonged exposure to digital screens may cause serious health problems in children. Weight gain as a result of unhealthy nutrition habits and inadequate physical activity, a predisposition to the development of metabolic syndrome, musculoskeletal disorders, sleeping disorders, psychiatric problems and behavioural disorders may develop (3-5). In addition, there were some concerns that light sources which emit the blue light might affect the anterior and posterior segment structures of the eye (6-8). To our knowledge, this is the first study in children to comprehensively investigate the effects of STC overuse arising from the restrictions during the COVID-19 pandemic on the ocular surface, anterior and posterior segment structures together.

\section{Materials and Methods}

This study was performed with the approval of Tepecik Training and Research Hospital's Medical Research Ethical Committee (approval number: 2020/10-19) and in line with ethical principles of the Declaration of Helsinki. Detailed information about the study was given to the children and their parents, and the risks were explained. Written informed consent forms were received from the participants and their parents.

Seventy-two children aged 10-18 years, using STC for at least two years, and routinely maintaining a screen distance of approximate $20-60 \mathrm{~cm}$ were initially included in the study. The data of 64 cases attending the check-up examinations were evaluated. Children having ocular trauma or surgery, cases with a history of significant refractive errors in their parents, contact lens wearers, those having chronic ocular disease and/or systemic disease capable of affecting the ocular structures, and children with an initial spherical equivalent refraction (SER) outside the range of -1.75 dioptres (D) to $+1.75 \mathrm{D}$ were not included in the study.

The best-corrected visual acuity (BCVA) was obtained by means of a Snellen chart. Ocular structures were evaluated with a slit-lamp biomicroscope and $90 \mathrm{D}$ lens. Intraocular pressure was measured by a Goldmann applanation tonometer. Cyclopentolate hydrochloride 1\% (Sikloplejin R; Abdi Ibrahim Company, Turkey) was applied three times with 10-minute intervals for cycloplegia. Axial length (AL), central corneal thickness (CCT), keratometry, anterior chamber depth (ACD), lens thickness (LT) and refraction were measured by the optical biometry (LenStar LS900, Haag-Streit Diagnostic, Switzerland) and autorefractor/ keratometry (Topcon KR-1, Japan) devices. Retinal nerve fibre layer (RNFL) thicknesses of the central, superior-temporal, superior-nasal, nasal, inferior-nasal, inferior-temporal and temporal sections were automatically determined by means of an optical coherence tomography (OCT) device (Heidelberg Spectralis; Heidelberg Engineering, Germany). Choroidal thicknesses were measured from the central area, $500 \mu \mathrm{m}$ nasal and $500 \mu \mathrm{m}$ temporal quadrants by means of an OCT device in enhanced-depth imaging mode. The vertical distance between the first (Bruch's membrane) and the second (internal surface of the sclera) hyperreflective lines was used for choroidal thickness measurements (9). Macular thicknesses were automatically measured from 1 $\mathrm{mm}$ diameter central (foveal) area, $3 \mathrm{~mm}$ diameter inner (parafoveal) area and $6 \mathrm{~mm}$ diameter outer (perifoveal) area by means of an OCT device. The inner and outer macular areas were further divided into four quadrants (superior, temporal, inferior, nasal). Measurements of nine subareas were recorded. Only the right eyes of the children were included in the study. All measurements were made by the same "blinded" researcher between 09.00-11.00 a.m. The average value of the three measurements was recorded. The measured cycloplegic refraction was converted to SER by means of the formula "sphere power $+1 / 2$ cylinder power". Myopia was defined as SER $<-0.50 \mathrm{D}$, hyperopia as SER> $+0.50 \mathrm{D}$ and emmetropia as $-0.50 \leq \mathrm{SER} \leq+0.50 \mathrm{D}$.

Tear film break-up time (TBUT) and Schirmer test with anaesthesia were performed for dry-eye evaluation. In TBUT test, fluorescein strip (Fluorescein paper, Haag-Streit AG, Switzerland) was moistened with saline solution and touched to the lower fornix. Cases were told to keep their eyes open until the first dry spots were seen on the tear film under illumination with cobalt blue light. The time interval between the last blink and the formation of the first dry spots was measured. In Schirmer test with anaesthesia, proparacaine $\mathrm{HCl}$ 0.5\% (Alcain; Alcon Company, Belgium) was administered, followed by a 5 minute wait. Schirmer test paper (SNO strips, Laboratory Chauvin, France) was placed on $1 / 3$ outer edge of the lower eyelid. After 5 minutes, the amount of wetting on the paper was recorded (10). In research, the tear-film stability was mainly determined by means of TBUT test, while secretion and the amount of the tear film were measured by means of Schirmer test (11). In individuals, the presence, frequency and severity of dry-eye symptoms were determined by means of the ocular surface disease index (OSDI) questionnaire (10). In our study, unanswered questions due to unperformed activities in the OSDI questionnaire were not included in the scoring. The sum of valid answers was multiplied by 25 , and the result was divided by the number of valid questions. Thus, the total OSDI score was determined. The children and their 
parents were asked to keep a diary showing their STC use time and outdoor activity time for 2 weeks. Daily STC use time (DSTCUT) was calculated by dividing the total STC use time by 14 . Daily outdoor activity time was calculated similarly. The clinical findings and ocular parameters at baseline (the early periods of the pandemic) (group 1) were compared with those after 1 year (group 2). Additionally, correlations between DSTCUT and ocular parameters were evaluated in group 2. All cases in group 2 had excessive DSTCUT for at least 6 months.

\section{Statistical Analysis}

Statistical analysis was made with the IBM-SPSS version 20 program. Continuous variables were expressed as mean \pm standard deviation (minimum - maximum) values. The Kolmogorov-Smirnov test was used to examine whether the variables complied with normal distribution in groups. Comparisons of the groups were made by the paired sample t-test. Pearson correlation analysis was used to evaluate the effects of DSTCUT on ocular parameters. $\mathrm{P}<0.05$ was regarded as statistically significant.

\section{Results}

The mean age of the children was $13.15 \pm 2.03$ years initially. There were 34 (53.1\%) males and 30 (46.9\%) females. The BCVAs of all of the children were 20/20. The DSTCUT of group 2 was longer than that of group 1 $(5.62 \pm 0.57$ vs $1.49 \pm 0.33$ hours, $p<0.001)$. Compared to group 1, group 2 had significantly lower TBUT (12.70 \pm 1.45 vs $9.50 \pm 2.10$ seconds, $p=0.015)$, a higher OSDI score $(14.52 \pm 4.19$ vs $25.22 \pm 5.75, p=0.007)$, more myopic SER $(-1.00 \pm 0.30$ vs $-1.80 \pm 0.40 \mathrm{D}, \mathrm{p}=0.031)$, and greater $A L(22.69 \pm 0.43$ vs $23.42 \pm 0.37 \mathrm{~mm}, \mathrm{p}=0.038)$. Daily outdoor activity times, Schirmer test values, CCTs, keratometries, ACDs, LTs, RNFL thicknesses of all sections, central, nasal and temporal choroidal thicknesses, and macular thicknesses of all areas were similar in both groups ( $p>0.05)$. The clinical findings and ocular parameters of the groups are shown in Tables I and II.

In group 2, as DSTCUT increased, TBUT ( $r=-0.368$, $p=0.034)$ decreased, OSDI score $(r=0.384, p=0.028)$ increased and more myopic SER $(r=0.340, p=0.045)$ occurred. On the other hand, Schirmer test value, $A L$, $C C T$, keratometry value, ACD, LT, central RNFL thickness, central choroidal thickness and central macular thickness were not significantly correlated with DSTCUT in group 2 ( $p>0.05)$. The correlation results between DSTCUT and ocular parameters in group 2 are shown in Table III.

\section{Discussion}

As the duration of the pandemic extends, due to restrictions, children might make a habit of overusing STC compared to the early periods of the pandemic. In our study, group 2 had significantly longer DSTCUT compared to group 1. We thought that the distance education being applied more effectively over the course of time and the increasing use of STC for entertainment purposes at home might be responsible for this situation. Digital devices may affect the tear-film layer and ocular surface (12-14). In a cross-sectional study, Moon et al. (12) stated that children using a smartphone for approximate 3 hours daily had lower

\begin{tabular}{|c|c|c|c|}
\hline $\begin{array}{l}\text { Clinical findings and anterior segment } \\
\text { parameters }\end{array}$ & $\begin{array}{l}\text { Group } 1 \text { (Findings at baseline) } \\
\text { Mean } \pm \text { SD (range) }\end{array}$ & $\begin{array}{l}\text { Group } 2 \text { (Findings after } 1 \text { year) } \\
\text { Mean } \pm \text { SD (range) }\end{array}$ & $\mathrm{p}^{\mathrm{a}}$ \\
\hline Daily STC use time (hour) & $1.49 \pm 0.33(1-3)$ & $5.62 \pm 0.57(4-8)$ & $<0.001^{*}$ \\
\hline Daily outdoor activity time (hour) & $1.32 \pm 0.53(0.5-3)$ & $1.29 \pm 0.61(0.5-3)$ & 0.539 \\
\hline TBUT (second) & $12.70 \pm 1.45(10-15)$ & $9.50 \pm 2.10(7-14)$ & $0.015^{*}$ \\
\hline Schirmer test (millimeter) & $12.28 \pm 1.76(10-15)$ & $11.37 \pm 2.04(9-15)$ & 0.106 \\
\hline Total OSDI score & $14.52 \pm 4.19(9-22)$ & $25.22 \pm 5.75(18-40)$ & $0.007^{*}$ \\
\hline SER (diopter) & $-1.00 \pm 0.30(-1.50 /+0.25)$ & $-1.80 \pm 0.40(-2.50 /-0.25)$ & $0.031^{*}$ \\
\hline Axial length (millimeter) & $22.69 \pm 0.43(22.14-23.21)$ & $23.42 \pm 0.37(22.95-23.82)$ & $0.038^{*}$ \\
\hline Central corneal thickness (micrometer) & $541.74 \pm 17.28(519-564)$ & $542.23 \pm 15.74(521-562)$ & 0.893 \\
\hline Keratometry value (diopter) & $42.65 \pm 1.35(40.50-44.25)$ & $42.70 \pm 1.45(40.25-44.50)$ & 0.704 \\
\hline Anterior chamber depth (millimeter) & $3.65 \pm 0.17(3.38-3.84)$ & $3.64 \pm 0.19(3.36-3.89)$ & 0.735 \\
\hline Lens thickness (millimeter) & $3.39 \pm 0.21(3.11-3.68)$ & $3.36 \pm 0.24(3.07-3.74)$ & 0.587 \\
\hline
\end{tabular}


Table II. Posterior segment parameters of the groups

\begin{tabular}{|c|c|c|c|}
\hline Posterior segment parameters & $\begin{array}{l}\text { Group } 1 \text { (Findings at baseline) } \\
\text { Mean } \pm \text { SD (range) }\end{array}$ & $\begin{array}{l}\text { Group } 2 \text { (Findings after } 1 \text { year) } \\
\text { Mean } \pm \text { SD (range) }\end{array}$ & $p^{a}$ \\
\hline \multicolumn{4}{|l|}{ Retinal nerve fiber layer thickness } \\
\hline Central ( $\mu \mathrm{m})$ & $104.5 \pm 6.3(91-115)$ & $102.9 \pm 5.7(86-112)$ & 0.602 \\
\hline Superior-temporal ( $\mu \mathrm{m})$ & $108.2 \pm 13.6(92-128)$ & $109.4 \pm 17.3(85-132)$ & 0.714 \\
\hline Superior-nasal ( $\mu \mathrm{m})$ & $105.3 \pm 14.2(86-125)$ & $107.1 \pm 16.9(88-129)$ & 0.578 \\
\hline Nasal ( $\mu \mathrm{m})$ & $83.7 \pm 11.6(63-96)$ & $82.8 \pm 9.4(67-94)$ & 0.805 \\
\hline Inferior-nasal ( $\mu \mathrm{m})$ & $104.1 \pm 16.9(81-124)$ & $103.0 \pm 14.2(83-120)$ & 0.746 \\
\hline Inferior-temporal $(\mu \mathrm{m})$ & $102.8 \pm 10.4(89-117)$ & $104.7 \pm 15.3(81-123)$ & 0.527 \\
\hline Temporal ( $\mu \mathrm{m})$ & $94.9 \pm 9.1(79-112)$ & $92.6 \pm 12.4(76-114)$ & 0.489 \\
\hline \multicolumn{4}{|l|}{ Choroidal thickness } \\
\hline Central ( $\mu \mathrm{m})$ & $301.6 \pm 23.4(275-332)$ & $290.8 \pm 27.2(245-327)$ & 0.071 \\
\hline Nasal ( $\mu \mathrm{m})$ & $287.9 \pm 26.5(253-319)$ & $275.7 \pm 29.1(223-308)$ & 0.065 \\
\hline Temporal ( $\mu \mathrm{m})$ & $294.8 \pm 29.2(262-328)$ & $286.3 \pm 25.7(252-316)$ & 0.084 \\
\hline \multicolumn{4}{|l|}{ Macular thickness } \\
\hline Central area $(\mu \mathrm{m})$ & $276.2 \pm 46.7(224-329)$ & $274.5 \pm 42.9(226-325)$ & 0.419 \\
\hline \multicolumn{4}{|l|}{ Inner segment $(\mu \mathrm{m})$} \\
\hline Superior & $342.5 \pm 50.3(287-395)$ & $341.6 \pm 44.5(291-389)$ & 0.503 \\
\hline Temporal & $334.6 \pm 59.7(271-402)$ & $336.8 \pm 52.9(279-397)$ & 0.375 \\
\hline Inferior & $341.2 \pm 48.3(287-396)$ & $340.1 \pm 45.6(289-391)$ & 0.487 \\
\hline Nasal & $344.9 \pm 39.5(298-389)$ & $346.9 \pm 41.2(297-394)$ & 0.392 \\
\hline \multicolumn{4}{|l|}{ Outer segment $(\mu \mathrm{m})$} \\
\hline Superior & $293.5 \pm 56.8(224-354)$ & $293.0 \pm 52.3(232-349)$ & 0.624 \\
\hline Temporal & $292.4 \pm 49.5(235-346)$ & $290.8 \pm 47.2(237-342)$ & 0.428 \\
\hline Inferior & $284.7 \pm 42.8(237-335)$ & $286.8 \pm 45.7(235-339)$ & 0.380 \\
\hline Nasal & $354.2 \pm 35.1(309-392)$ & $354.9 \pm 38.6(308-397)$ & 0.583 \\
\hline
\end{tabular}

TBUTs compared to those using it for less than 1 hour daily. They also showed that there was a significant increase in TBUT in children with dry-eye when smartphone usage was discontinued for 4 weeks (12). Wu et al. (14) found that computer workers with a daily digital screen time of more than 4 hours had significantly shorter TBUTs compared to those with the daily digital screen time of 4 hours or less. The authors detected that the Schirmer scores of both groups were similar (14). On the other hand, Nakamura et al. (15) stated that, in office workers, the duration of computer use did not affect TBUT measurement. They reported that office workers using a computer for more than 8 hours per day had significantly lower Schirmer scores compared to those using it for less than 2 hours per day (15). In our study, the TBUT value was significantly lower in group 2 compared to group 1, while the Schirmer scores of the groups were similar. Additionally, as DSTCUT increased in group 2, the TBUT value decreased significantly. We considered that STC overuse during the pandemic might affect tear-film evaporation, and might increase the tendency of dry-eye in children. Blue-light exposure of the eyes may be responsible for this situation. One experimental study showed that overexposure to blue light increased the inflammatory markers and reactive oxygen species (ROS) on the ocular surface, disrupted the tear-film content, and thus caused dry-eye (6). In another study, computer workers using digital screens for a long time daily were determined to have worse meibomian gland functions compared to those 
Table III. The correlation results between daily smartphone, tablet and computer use time and ocular parameters in group 2

\begin{tabular}{|c|c|c|}
\hline & \multicolumn{2}{|c|}{$\begin{array}{l}\text { DSTCUT of } \\
\text { group } 2\end{array}$} \\
\hline & $\mathbf{r}$ & p-value \\
\hline Tear beak-up time & -0.368 & $0.034^{*}$ \\
\hline Schirmer test value & -0.265 & 0.073 \\
\hline Ocular surface disease index score & 0.384 & $0.028^{*}$ \\
\hline Spherical equivalent refraction & 0.340 & $0.045^{*}$ \\
\hline Axial length & 0.296 & 0.059 \\
\hline Central corneal thickness & 0.046 & 0.795 \\
\hline Keratometry value & 0.075 & 0.592 \\
\hline Anterior chamber depth & -0.161 & 0.323 \\
\hline Lens thickness & -0.133 & 0.409 \\
\hline Central retinal nerve fiber layer thickness & 0.107 & 0.568 \\
\hline Central choroidal thickness & -0.263 & 0.081 \\
\hline Central macular thickness & 0.143 & 0.377 \\
\hline
\end{tabular}

using them for a short time daily (14). Meibomian gland dysfunction might cause a reduction in lipid secretion, an increase in tear evaporation, and thus the development of dry-eye $(14,16,17)$.

In clinical practice, OSDI score is used to show the severity of dry-eye symptoms (10). Moon et al. (12) reported that smartphone use in children caused an increase in their OSDI score. The authors also showed that there was a significant decrease in the OSDI score in children with dry-eye when their smartphone use was discontinued for 4 weeks (12). Computer workers with a daily digital screen time of more than 4 hours were detected to have significantly higher OSDI scores compared to those with the daily digital screen time of 4 hours or less (14). In our study, OSDI score was significantly higher in group 2 compared to group 1. Additionally, as DSTCUT increased in group 2, OSDI score increased significantly. We thought that STC overuse during the pandemic might increase dry-eye complaints in children.

Increased usage of digital screens may play a role in the development of temporary or permanent myopia (18-21). Liu et al. (22) found that children having greater myopic refraction spent more time using computers and smartphones. Myopia development was reported to be more frequent in children with a digital screen contact time of more than 2 hours daily (20). Similarly, Hansen et al. (19) determined that children using digital screens for more than 6 hours daily had a higher risk of developing myopia compared to those using them for less than 2 hours daily. We detected that group 2 had significantly more myopic SER and greater AL compared to group 1. Additionally, as DSTCUT increased in group 2, more myopic SER occurred. In the literature, children spending more time on computers and smartphones were stated to have longer $\operatorname{AL}(22,23)$. In children, annual SER changes towards myopia were found between 0.16-0.55 D (24-26), while annual axial elongations were reported between $0.22-0.35 \mathrm{~mm}$ (25-27). In our study, annual SER and AL changes during the pandemic were higher than the values reported in the literature, and these increases might be associated with STC overuse. Closer working and excessive accommodation, a higher "accommodative convergence/accommodation" ratio, or peripheral defocus are among the possible mechanisms blamed in the development and progression of myopia due to digital device usage (28). Prolonged digital device usage at a close distance may cause blurred images on the retina due to hyperopic defocus, and the retina may send remodelling signals to the sclera. As a result of this, axial elongation might occur (29).

In our study, CCTs, keratometries, ACDs and LTs of the two groups were similar. Additionally, CCT, keratometry value, $A C D$ and $L T$ were not significantly correlated with DSTCUT in group 2. Therefore, we thought that at 1-year follow-up, STC overuse during the pandemic had no effect on CCT, keratometry value, ACD and LT. Similarly, Liu et al. (22) determined that the values of CCT, ACD and LT were not significantly associated with the time spent on different digital devices. In the literature, there were some concerns that blue light emitted from digital screens might affect the posterior segment structures $(7,8)$. In experimental cell and rat models, it was reported that periodic exposure to smartphone-mimicking low-luminance blue light might cause retinal damage by means of Bcl-2/BAX-dependent apoptosis (7). In another experimental study, it was stated that low-intensity blue light from digital devices, such as monitors and smartphones, might induce ROS production and might cause apoptosis in retinal pigment epithelial cells (8). However, in our study, the RNFL thicknesses of all sections, central, nasal and temporal choroidal thicknesses, and the macular thicknesses of all areas had not changed significantly at 1-year follow-up. Perhaps prolonged STC usage for many years may be necessary for the occurrence of the posterior segment involvement in clinical practice. 


\section{Study Limitations}

Our study had some limitations. There might be other undetectable additional factors predisposing to dry-eye and myopia or affecting their development. In addition, the children and their parents answering the questionnaire and keeping the timeline might have assumed a relationship between STC usage and ocular findings. Despite these limitations, we believe that this study can provide valuable and comprehensive information about the effects of STC overuse during the COVID-19 pandemic on ocular surface, anterior and posterior segment structures.

\section{Conclusion}

In summary, we found that STC overuse during the pandemic might increase the tendency to dry-eye and myopia, and might cause axial elongation. We also determined that at 1-year follow-up, STC overuse had no effect on CCT, keratometry, ACD, LT, and posterior segment parameters. Reducing digital screen contact time during a pandemic may be beneficial in preventing the development or progression of these findings.

\section{Ethics}

Ethics Committee Approval: This study was performed with the approval of Tepecik Training and Research Hospital's Medical Research Ethical Committee (approval number: 2020/10-19).

Informed Consent: Written informed consent forms were received from the participants and their parents.

Peer-review: Externally peer-reviewed.

\section{Authorship Contributions}

Concept: H.Ö., B.Ö., Design: H.Ö., B.Ö., Data Collection or Processing: B.Ö., Analysis or Interpretation: H.Ö., B.Ö., Writing: H.Ö.

Conflict of Interest: No conflict of interest was declared by the authors.

Financial Disclosure: The authors declared that this study received no financial support.

\section{References}

1. Rosenfield M, Howarth PA, Sheedy JE, Crossland MD. Vision and IT displays: a whole new visual world. Ophthalmic Physiol Opt 2012; 32:363-6.

2. Council on Communications and Media. Media use in schoolaged children and adolescents. Pediatrics 2016; 138:e20162592. doi: 10.1542/peds.2016-2592.

3. Bedford $\mathrm{R}$, Saez de Urabain IR, Cheung $\mathrm{CH}$, Karmiloff-Smith A, Smith TJ. Toddlers' fine motor milestone achievement is associated with early touchscreen scrolling. Front Psychol 2016; 7:1108
4. Touitou Y, Touitou D, Reinberg A. Disruption of adolescents' circadian clock: The vicious circle of media use, exposure to light at night, sleep loss and risk behaviors. J Physiol Paris 2016; 110:467-79.

5. Mylona I, Deres ES, Dere GS, Tsinopoulos I, Glynatsis M. The impact of internet and videogaming addiction on adolescent vision: A review of the literature. Front Public Health 2020; 8:63.

6. Lee HS, Cui L, Li Y, et al. Influence of light emitting diode-derived blue light overexposure on mouse ocular surface. PLoS One 2016; 11:e0161041. doi: 10.1371/journal.pone.0161041.

7. Lin $\mathrm{CH}, \mathrm{Wu} M \mathrm{MR}, \mathrm{Li} \mathrm{CH}$, et al. Editor's highlight: periodic exposure to smartphone-mimic low-luminance blue light induces retina damage through bcl-2/bax-dependent apoptosis. Toxicol Sci 2017; 157:196-210.

8. Moon J, Yun J, Yoon YD, et al. Blue light effect on retinal pigment epithelial cells by display devices. Integr Biol (Camb) 2017; 9:436-43.

9. Boonarpha $N$, Zheng $\mathrm{Y}$, Stangos $\mathrm{AN}$, et al. Standardization of choroidal thickness measurements using enhanced depth imaging optical coherence tomography. Int J Ophthalmol 2015; 8:484-91.

10. Wolffsohn JS, Arita R, Chalmers R, et al. TFOS DEWS II Diagnostic Methodology report. Ocul Surf 2017; 15:539-74.

11. Jaiswal S, Asper L, Long I, Lee A, Harrison K, Golebiowski B. Ocular and visual discomfort associated with smartphones, tablets and computers: what we do and do not know. Clin Exp Optom 2019; 102:463-77.

12. Moon JH, Kim KW, Moon NJ. Smartphone use is a risk factor for pediatric dry eye disease according to region and age: a case control study. BMC Ophthalmol 2016; 16:188.

13. Moon JH, Lee MY, Moon NJ. Association between video display terminal use and dry eye disease in school children. I Pediatr Ophthalmol Strabismus 2014; 51:87-92.

14. $\mathrm{Wu} \mathrm{H}$, Wang $\mathrm{Y}$, Dong $\mathrm{N}$, et al. Meibomian gland dysfunction determines the severity of the dry eye conditions in visual display terminal workers. PLoS One 2014; 9:e105575. doi: 10.1371/ journal.pone.0105575.

15. Nakamura S, Kinoshita S, Yokoi N, et al. Lacrimal hypofunction as a new mechanism of dry eye in visual display terminal users. PLoS One 2010; 5:e11119. doi: 10.1371/journal.pone.0011119.

16. Fenga C, Aragona P, Cacciola A, et al. Meibomian gland dysfunction and ocular discomfort in video display terminal workers. Eye (Lond) 2008; 22:91-5.

17. Pult $\mathrm{H}$, Riede-Pult $\mathrm{BH}$, Nichols IJ. Relation between upper and lower lids' meibomian gland morphology, tear film, and dry eye. Optom Vis Sci 2012; 89:E310-5. doi: 10.1097/ OPX.0b013e318244e487. Erratum in: Optom Vis Sci 2012; 89:517.

18. Enthoven CA, Tideman JWL, Polling IR, Yang-Huang J, Raat $\mathrm{H}$, Klaver CCW. The impact of computer use on myopia development in childhood: the Generation R study. Prev Med 2020; 132:105988.

19. Hansen $\mathrm{MH}$, Laigaard PP, Olsen EM, et al. Low physical activity and higher use of screen devices are associated with myopia at the age of 16-17 years in the CCC2000 eye study. Acta Ophthalmol 2020; 98:315-21.

20. Singh NK, James RM, Yadav A, Kumar R, Asthana S, Labani $\mathrm{S}$. Prevalence of myopia and associated risk factors in schoolchildren in North India. Optom Vis Sci 2019; 96:200-5. 
21. Ciuffreda KJ, Vasudevan B. Nearwork-induced transient myopia (NITM) and permanent myopia--is there a link? Ophthalmic Physiol Opt 2008; 28:103-14.

22. Liu S, Ye S, Xi W, Zhang X. Electronic devices and myopic refraction among children aged 6-14 years in urban areas of Tianjin, China. Ophthalmic Physiol Opt 2019; 39:282-93.

23. Terasaki H, Yamashita T, Yoshihara N, Kii Y, Sakamoto T. Association of lifestyle and body structure to ocular axial length in Japanese elementary school children. BMC Ophthalmol 2017; 17:123.

24. French AN, Morgan IG, Burlutsky G, Mitchell P, Rose KA. Prevalence and 5- to 6-year incidence and progression of myopia and hyperopia in Australian schoolchildren. Ophthalmology 2013; 120:1482-91.

25. You X, Wang L, Tan $H$, et al. Near work related behaviors associated with myopic shifts among primary school students in the jiading district of shanghai: a school-based one-year cohort study. PLoS One 2016; 11:e0154671. doi: 10.1371/journal. pone. 0154671 .

26. Ma Y, Zou H, Lin S, et al. Cohort study with 4-year follow-up of myopia and refractive parameters in primary schoolchildren in Baoshan District, Shanghai. Clin Exp Ophthalmol 2018; 46:86172.

27. Wang D, Ding X, Liu B, Zhang J, He M. Longitudinal changes of axial length and height are associated and concomitant in children. Invest Ophthalmol Vis Sci 2011; 52:7949-53.

28. Mccrann S, Loughman J, Butler JS, Paudel N, Flitcroft DI. Smartphone use as a possible risk factor for myopia. Clin Exp Optom 2021; 104:35-41.

29. Honda T, Nakagawa T, Watanabe Y, et al. Association between information and communication technology use and ocular axial length elongation among middle-aged male workers. Sci Rep 2019; 9:17489. 